\section{A computer program \\ for restricted oblique Procrustes rotation to a primary factor pattern matrix}

\author{
GEORGE J. HUBA \\ Yale University, New Haven, Connecticut 06520
}

Among the most important problems in factor analytic research is the demonstration that a factor matrix is equivalent to either a previously obtained or theoretically meaningful factor matrix. A class of rotations, known as Procrustean transformations, has evolved for rotating a factor matrix (A) to a position where the columns of $A$ are most similar to those of some target factor matrix (B).

The general Procrustes rotation problem can be stated as follows. Let $\mathrm{A}$ and $\mathrm{B}$ be $\mathrm{n}$ by $\mathrm{p}$ factor matrices (pattern, structure, or reference structure) where $n>p$. In order to rotate $A$ to a position of greatest similarity to $B$, it is necessary to find a $\mathrm{p}$ by $\mathrm{p}$ transformation matrix (T) such that

$$
\operatorname{tr}\left[(\mathrm{B}-\mathrm{AT})^{\circ}(\mathrm{B}-\mathrm{AT})\right]
$$

is minimized. Different restrictions are placed on the matrix $T$ depending on whether $A$ and $B$ are factor pattern, factor structure, or reference structure matrices, and whether the rotation is oblique or orthogonal. In the case of oblique Procrustean transformations to a factor pattern matrix, unit length for the rotated factors represented by the matrix produce $A T$ may be maintained by placing $T$ under the restraint that

$$
\operatorname{diag}\left(\mathrm{T}^{\prime} \mathrm{T}\right)^{-1}=\mathrm{I}
$$

where $I$ is a $p$ by $p$ identity matrix.

A widely circulated computer program (Hurley \& Cattell, 1962) performs an approximate oblique Procrustes rotation to a reference structure matrix. Algorithms have been presented by Browne and Kristof (1969), Gruvaeus (1970), and Browne (1972b) for exact oblique Procrustes transformations to a primary factor pattern matrix. The method presented by Browne and Kristof (1969) has been shown by Gruvaeus (1970) to be sensitive to singularity, and tests by the present writer have demonstrated that Browne and Kristof's method gives erroneous results in a fairly large percentage of real data rotations. The methods of Gruvaeus (1970) and Browne (1972b) present an advance over the earlier method of Browne and Kristof (1969) not only in computational accuracy, but also because not all elements of the target matrix must be specified (fixed) in these algorithms. The present program uses the algorithm of Browne (1972b) which is a special case of the direct factor pattern rotation method suggested by Jennrich and Sampson (1966). Browne (1972b) has shown that his method finds a smaller minimization of Equation 1 than the algorithm of Gruvaeus (1970) under certain conditions.

Computational procedure. The algorithm uses a modification of the computational procedure suggested by Jennrich and Sampson (1966). Factors are rotated two at a time. For each pair of factors a Newton-Raphson iterative minimization procedure is used to determine the angle of the rotation, and after each rotation the correlation matrix among factors is updated. Cycles of the rotation of all possible pairs of factors are made until no further rotation (within a specified convergence criterion) is required. Before each cycle of rotations, the program sorts the columns of the matrix being rotated and the correlation matrix among factors using the algorithm suggested by Browne (1972a). The column sort results in decreased computing time and increased accuracy. In the first three cycles of the rotation procedure, very extensive calculations are made to determine starting estimates for the Newton-Raphson minimization. Experience with the program has shown that after three iterations an initial value of zero is adequate for fast convergence.

Input. Identifying information input to the program includes a problem title, a format card, and a parameter card stating the number of variables, the number of factors, whether the original factor matrix is orthogonal, the number of specified elements in each column of the target matrix, and the elements in each column of the target matrix which are fixed. Then the initial factor matrix, the correlations among the initial factors (if the factors are not orthogonal), and the target factor pattern matrix are input under the format specified.

Output. All input information is initially printed. Then the matrix is rotated and the minimization found for Equation 1 is printed after each cycle. Finally, the rotated matrix and the resulting correlations among factors are printed. In addition, in those cases where the entire target matrix has been specified, the following calculations are made: (1) Kaiser's (1974) index of factorial simplicity for the target matrix, the unrotated factor matrix, and the rotated factor matrix; (2) Tucker's factor similarity coefficients (cf. Harmon, 1967) between the target matrix and the unrotated factor matrix; (3) Tucker's factor similarity coefficients between the target matrix and the rotated factor matrix; (4) the matrix B - AT; (5) the matrix (B - AT) ' (B - AT).

Language and computer. The program is written in FORTRAN IV and has been compiled using the $\mathrm{G}$ and Hextended compilers on an IBM 370/158. Under the H-extended compiler, compilation time has been approximately $6 \mathrm{sec}$ and the rotation of a 12 by 4 matrix to a fully specified target matrix has taken less than $1 \mathrm{sec}$. The program is currently dimensioned for 35 variables and 10 factors and requires $58 \mathrm{~K}$ bytes.

Availability. A copy of this paper, a source listing, a manual for the program, and test results are available from George J. Huba, Yale Psychology Department, 2 Hillhouse Avenue, New Haven, Connecticut 06520. Source and test data decks are available at the cost of duplication and postage.

\section{REFERENCES}

Browne, M. W. Orthogonal rotation to a partially specified target. British Journal of Mathematical and Statistical Psychology, 1972, 25, 115-120. (a)

Browne, M. W. Oblique rotation to a partially specified target. British Journal of Mathematical and Statistical Psychology, 1972, 25, 207-212. (b)

Browne, M. W., \& Kristof, $W$. On the oblique rotation of a factor matrix to a specified pattern. Psychometrika, 1969, 34, 237-248.

Gruvaeus, G. T. A general approach to the Procrustes pattern rotation. Psy chometrika, 1970, 35, 493-505.

Harmon, H. H. Modern factor analysis. (Rev, ed.) Chicago: University of Chicago Press, 1967.

Hurley, J. R. \& Cattell, R. B. The Procrustes program, producing direct rotation to test a hypothesized factor structure. Behavioral Science, 1962, 7, 258-262.

Jennrich, R. I., \& Sampson, P. F. Rotation for simple loadings. Psychometrika, 1966, 31, 313-323.

Kaiser, H. F. An index of factorial simplicity. Psychometrika $1974,39,31-36$. 